\title{
The inverse eigenvalue and inertia problems for minimum rank two graphs
}

\section{Authors}

Wayne Barrett, Seth Gibelyou, Mark Kempton, Nicole Malloy, Curtis Nelson, William Sexton, and John Sinkovic 


\title{
THE INVERSE EIGENVALUE AND INERTIA PROBLEMS FOR MINIMUM RANK TWO GRAPHS*
}

\author{
WAYNE BARRETT ${ }^{\dagger}$, SETH GIBELYOU ${ }^{\ddagger}$, MARK KEMPTON ${ }^{\ddagger}$, NICOLE MALLOY \\ CURTIS NELSON ${ }^{\ddagger}$, WILLIAM SEXTON ${ }^{\ddagger}$, AND JOHN SINKOVIC ${ }^{\ddagger}$
}

\begin{abstract}
Let $G$ be an undirected graph on $n$ vertices and let $\mathcal{S}(G)$ be the set of all real symmetric $n \times n$ matrices whose nonzero off-diagonal entries occur in exactly the positions corresponding to the edges of $G$. Let $\operatorname{mr}(G)$ denote the minimum rank of all matrices in $\mathcal{S}(G)$, and $\mathrm{mr}_{+}(G)$ the minimum rank of all positive semidefinite matrices in $\mathcal{S}(G)$. All graphs $G$ with $\operatorname{mr}(G)=2$ and $\mathrm{mr}_{+}(G)=k$ are characterized; it is also noted that $\operatorname{mr}_{+}(G)=\alpha(G)$ for such graphs. This characterization solves the inverse inertia problem for graphs whose minimum rank is two. Furthermore, it is determined which diagonal entries are required to be zero, are required to be nonzero, or can be either for a rank minimizing matrix in $\mathcal{S}(G)$ when $\operatorname{mr}(G)=2$. Collectively, these results lead to a solution to the inverse eigenvalue problem for rank minimizing matrices for graphs whose minimum rank is two.
\end{abstract}

Key words. Combinatorial matrix theory, Inertia, Inverse eigenvalue problem, Inverse inertia problem, Graph, Minimum positive semidefinite rank, Minimum rank, Nil vertex, Symmetric.

AMS subject classifications. 05C50, 15A03, 15A, 15B57.

1. Introduction. One of the fundamental questions in combinatorial matrix theory is the Minimum Rank Problem, which asks: what is the minimum rank of all real symmetric $n \times n$ matrices with a given off-diagonal zero pattern? This question has also been considered for matrices with entries in any field and even for nonsymmetric matrices but in this study we are concerned with questions of possible inertias and sets of eigenvalues of real symmetric matrices. Any off-diagonal zero pattern of such matrices can be encoded in a graph by taking a set of $n$ vertices and constructing edges corresponding to the nonzero off-diagonal entries. Conversely, there is an infinite set of such matrices corresponding to the pattern encoded in a graph $G$, which we denote by $\mathcal{S}(G)$. The smallest rank attained by a matrix in $\mathcal{S}(G)$ is the minimum rank of $G$, denoted $\operatorname{mr}(G)$. Though far from solved, many results have been published that help to answer this question. In particular, Barrett, van der Holst, and Loewy [4] classified

${ }^{*}$ Received by the editors on August 14, 2010. Accepted for publication on March 11, 2011. Handling Editor: Raphael Loewy.

$\dagger^{\dagger}$ Department of Mathematics, Brigham Young University, Provo, Utah 84602, USA (wayne@math.byu.edu).

${ }^{\ddagger}$ Brigham Young University, Provo, Utah 84602, USA (tigerbot_1@hotmail.com, mark.kempton@gmail.com, nicolea.malloy@gmail.com, curtisgn@gmail.com, wnsexton@gmail.com, johnsinkovic@gmail.com). 
all graphs with minimum rank 2 . The current paper provides a deeper characterization of graphs $G$ with minimum rank 2 by examining their minimum positive semidefinite rank, the minimum rank of all positive semidefinite matrices in $\mathcal{S}(G)$. For a graph $G$ such that $\operatorname{mr}(G)=2$ and $\mathrm{mr}_{+}(G)=k$, we present equivalent statements concerning the complement of $G$, forbidden subgraphs of $G$ and the independence number of $G$. One result of this characterization is that the minimum positive semidefinite rank of a graph with minimum rank 2 can be found by computing the independence number. Although in general this is difficult, the independence number for this class of graphs is easily computable.

One generalization of the Minimum Rank Problem is the Inverse Eigenvalue Problem, which asks what eigenvalues are possible for a matrix with a given off-diagonal zero pattern? We present a solution to the Inverse Eigenvalue Problem for rank minimizing matrices for graphs whose minimum rank is two. Our result bears some similarity to what is reported in [1] as will be discussed near the beginning of Section 6.

A generalization of the Minimum Rank Problem that is also a simplification of the Inverse Eigenvalue Problem is the Inverse Inertia Problem. The inertia of a matrix is an ordered triple giving the number of positive eigenvalues, the number of negative eigenvalues, and the multiplicity of the eigenvalue 0 for that matrix. Without losing any information we can drop the multiplicity of 0 to obtain an ordered pair which we call the partial inertia. The Inverse Inertia Problem consists of classifying all possible partial inertias for matrices in $\mathcal{S}(G)$. Due to results from [3], the partial inertias of graphs with minimum rank 2 are completely classified by knowing $\operatorname{mr}_{+}(G)$. Thus, the characterization above solves the Inverse Inertia Problem for graphs with minimum rank 2 .

A third generalization that has not been widely studied considers the structure of matrices $A$ in $\mathcal{S}(G)$ for which $\operatorname{rank} A=\operatorname{mr}(G)$. We denote this subset of $\mathcal{S}(G)$ by $\mathscr{M} \mathscr{R}(G)$. Although this is a related problem to the Inverse Inertia and Eigenvalue Problems, its complexity is not easily comparable to theirs. Knowing details about the structure of $\mathscr{M} \mathscr{R}(G)$ can be helpful in certain aspects of the Inverse Eigenvalue Problem, and knowing the possible inertias for a graph can be helpful in determining the structure of $\mathscr{M} \mathscr{R}(G)$. In many cases $\mathscr{M} \mathscr{R}(G)$ is a sufficiently restricted subset that certain diagonal entries are required to be zero or nonzero for all matrices in $\mathscr{M} \mathscr{R}(G)$. We call the vertices corresponding to such diagonal entries respectively nil and nonzero vertices. Finding nil and nonzero vertices is a first step toward classifying $\mathscr{M} \mathscr{R}(G)$. For graphs with minimum rank 2 we classify all nil and nonzero vertices. Consequently, we also classify all vertices that are neither nil nor nonzero, which we call neutral vertices. 


\section{Definitions.}

\subsection{Matrix theory.}

Definition 2.1. Given a graph $G$ on $n$ vertices, let $\mathcal{S}(G)$ be the set of all real symmetric $n \times n$ matrices $A=\left[a_{i j}\right]$ such that $a_{i j} \neq 0, i \neq j$, if and only if $i j$ is an edge of $G$. Let $\mathcal{S}_{+}(G)$ be the subset of $\mathcal{S}(G)$ consisting of all positive semidefinite matrices in $\mathcal{S}(G)$. Then the minimum rank of $G$ is

$$
\operatorname{mr}(G)=\min _{A \in \mathcal{S}(G)}\{\operatorname{rank} A\}
$$

The minimum positive semidefinite rank of $G$ is

$$
\operatorname{mr}_{+}(G)=\min _{A \in \mathcal{S}_{+}(G)}\{\operatorname{rank} A\}
$$

Definition 2.2. Given a graph $G$, let

$$
\mathscr{M} \mathscr{R}(G)=\{A \in \mathcal{S}(G) \mid \operatorname{rank} A=\operatorname{mr}(G)\} .
$$

Definition 2.3. Given a matrix $A$ we define the inertia of $A$ as the triple $(\pi(A), \nu(A), \delta(A))$, where $\pi(A)$ denotes the number of positive eigenvalues of $A, \nu(A)$ the number of negative eigenvalues of $A$, and $\delta(A)$ the multiplicity of 0 as an eigenvalue of $A$.

We note that for an $n \times n$ matrix $A, \pi(A)+\nu(A)+\delta(A)=n$.

Definition 2.4. The partial inertia of a symmetric matrix $A$, denoted $\operatorname{pin}(A)$, is the ordered pair $(\pi(A), \nu(A))$ where $\pi$ and $\nu$ are as in Definition 2.3.

We note that for any symmetric matrix $A, \pi(A)+\nu(A)=\operatorname{rank} A$.

Definition 2.5. Given a graph $G$, the inertia set of $G$, denoted $\mathcal{I}(G)$, is the set of all possible partial inertias that can be obtained by matrices in $\mathcal{S}(G)$. That is

$$
\mathcal{I}(G)=\{(r, s) \in \mathbb{N} \times \mathbb{N} \mid \operatorname{pin}(A)=(r, s) \text { for some } A \in \mathcal{S}(G)\} .
$$

(Here we include the number 0 in $\mathbb{N}$.)

Definition 2.6. Let $m$ and $n$ be non-negative integers with $m \leq n$. When plotted as points in $\mathbb{R}^{2}$, the set

$$
\left\{(r, s) \in \mathbb{N}^{2} \mid m \leq r+s \leq n\right\}
$$

forms a trapezoid. We denote this set by $\mathrm{T}[m, n]$.

ObSERVATION 2.7. For any graph $G$ on $n$ vertices, $\mathcal{I}(G) \subseteq \mathrm{T}[\operatorname{mr}(G), n]$. 
Definition 2.8. Let $G$ be a graph on $n$ vertices. If $\mathcal{I}(G)=\mathrm{T}[k, n]$ for some nonnegative integer $k$, we say that $\mathcal{I}(G)$ is a trapezoid.

ObSERvation 2.9. If $\mathcal{I}(G)$ is a trapezoid, then $\mathcal{I}(G)=\mathrm{T}[\operatorname{mr}(G), n]$.

It is shown in [5] that for most graphs $G$ on six or fewer vertices, $\mathcal{I}(G)$ is a trapezoid.

\subsection{Graph theory.}

Definition 2.10. A clique in a graph is a set of vertices which are pairwise adjacent. A complete graph is a graph whose vertex set forms a clique. The complete graph on $n$ vertices is notated $K_{n}$.

Definition 2.11. The clique number of a graph $G$ denoted $\omega(G)$, is the size of the largest clique in $G$.

Definition 2.12. A set of vertices in a graph $G$ is an independent set if its vertices are pairwise non-adjacent. The independence number of $G$, denoted $\alpha(G)$, is the size of the largest independent set in $G$.

Definition 2.13. The complement of a graph $G=(V, E)$ is the graph $G^{c}=$ $\left(V, E^{c}\right)$, where $E^{c}$ consists of all two element sets from $V$ that are not in $E$.

We note that $\alpha(G)=\omega\left(G^{c}\right)$.

Definition 2.14. A vertex v in a graph $G$ is a

- nil vertex if its corresponding diagonal entry $d_{v}$ is zero in every matrix in $\mathscr{M} \mathscr{R}(G)$.

- nonzero vertex if its corresponding diagonal entry $d_{v}$ is nonzero in every matrix in $\mathscr{M} \mathscr{R}(G)$.

- neutral vertex if it is neither a nil vertex nor a nonzero vertex.

The following examples show the identification of nil, nonzero, and neutral vertices

Example: Let $S_{4}$ be the star on 4 vertices with $V=\{1,2,3,4\}$ and $E=\{12,13,14\}$. Every matrix in $\mathscr{M} \mathscr{R}\left(S_{4}\right)$ is of the form

$$
A=\left[\begin{array}{cccc}
d_{1} & a & b & c \\
a & d_{2} & 0 & 0 \\
b & 0 & d_{3} & 0 \\
c & 0 & 0 & d_{4}
\end{array}\right]
$$

where $d_{2}, d_{3}, d_{4}$ correspond to the pendant vertices of $S_{4}$. Since $\operatorname{mr}\left(S_{4}\right)=2, \operatorname{rank} A=$ 2. If any of $d_{2}, d_{3}$, or $d_{4}$ is not 0 , then rank $A$ is greater than 2 . Hence, every pendant vertex of $S_{4}$ is a nil vertex. 
Further, both $\left[\begin{array}{llll}1 & 1 & 1 & 1 \\ 1 & 0 & 0 & 0 \\ 1 & 0 & 0 & 0 \\ 1 & 0 & 0 & 0\end{array}\right]$ and $\left[\begin{array}{llll}0 & 1 & 1 & 1 \\ 1 & 0 & 0 & 0 \\ 1 & 0 & 0 & 0 \\ 1 & 0 & 0 & 0\end{array}\right]$ are in $\mathscr{M} \mathscr{R}\left(S_{4}\right)$ and thus $d_{1}$ is a neutral vertex.

Example: Consider $K_{n}, n \geq 2$. Let $A \in \mathscr{M} \mathscr{R}\left(K_{n}\right)$. Since $\operatorname{mr}\left(K_{n}\right)=1, \operatorname{rank} A=1$. If any diagonal entry of $A$ were zero, then the rank of $A$ would be at least two. Therefore, every diagonal entry of $A$ is nonzero and thus every vertex of $K_{n}$ is a nonzero vertex.

Definition 2.15. Given two graphs $G$ and $H$ with $V(G) \cap V(H)=\emptyset$, the union of $G$ and $H$ is the graph $(V(G) \cup V(H), E(G) \cup E(H))$ and is written $G \cup H$.

Definition 2.16. Given two graphs $G$ and $H$ with $V(G) \cap V(H)=\emptyset$, the join of $G$ and $H$, written $G \vee H$, is the graph with vertex set $V(G) \cup V(H)$ and edge set

$$
E(G) \cup E(H) \cup\{u v \mid u \in V(G) \text { and } v \in V(H)\} .
$$

Definition 2.17. A decomposable graph (or cograph) $G$ is defined recursively as follows:

- $K_{1}$ is decomposable.

- If $G_{1}$ and $G_{2}$ are decomposable, $G_{1} \cup G_{2}$ is decomposable.

- If $G_{1}$ and $G_{2}$ are decomposable, $G_{1} \vee G_{2}$ is decomposable.

Definition 2.18. A graph $G$ is said to be $H$-free if $H$ is not an induced subgraph of $G$.

\section{Previous results and lemmata.}

Theorem 3.1. [7] A graph $G$ is decomposable if and only if $G$ is $P_{4}$-free.

The following two results are Theorem 9 and Theorem 11 from [4].

TheOREM 3.2. Let $G$ be a connected graph and let $F$ be an infinite field with char $F \neq 2$. Then the following are equivalent:

1. $\operatorname{mr}(F, G) \leq 2$.

2. $G^{c}$ can be expressed as the union of at most 2 complete graphs and of complete bipartite graphs.

3. $G$ is $\left(P_{4}\right.$, dart, $\left.\ltimes, K_{3,3,3}\right)$-free.

THEOREM 3.3. Given a connected graph, $G$, the following are equivalent:

1. $m r_{+}(G) \leq 2$. 
2. $G^{c}$ is the union of complete bipartite graphs.

3. $G$ is $\left(K_{3}^{c}, P_{4}\right)$-free. $\left(G\right.$ is $P_{4}$-free with $\alpha(G) \leq 2$.)

We remark that it is well known that complete graphs on two or more vertices are the only connected graphs with minimum rank 1 .

The next result is Corollary 2.7 from [6] and the observation follows immediately from definitions.

TheOREm 3.4. For a connected graph $G, \alpha(G) \leq \mathrm{mr}_{+}(G)$.

OBservation 3.5. For a graph $G, \operatorname{mr}(G) \leq \mathrm{mr}_{+}(G)$.

The following two results are Lemma 1.1 and Theorem 7.1 from [3].

LEMMA 3.6. [Northeast Lemma] Let $G$ be a graph on $n$ vertices and suppose that $A \in \mathcal{S}(G)$ with $\operatorname{pin}(A)=(\pi, \nu)$. Then for every pair of integers $r \geq \pi$ and $s \geq \nu$ satisfying $r+s \leq n$, there exists a matrix $B \in \mathcal{S}(G)$ with $\operatorname{pin}(B)=(r, s)$.

THEOREM 3.7. Let $G$ be a graph and let $M \in \mathcal{S}(G)$ be a real symmetric matrix with partial inertia $(k, 0), k>1$. Then there exists a matrix $M^{\prime} \in \mathcal{S}(G)$ with partial inertia $(r, s)$ satisfying $r<k$ and $s<k$.

Corollary 3.8. Let $G$ be a graph and suppose that $(2,0) \in \mathcal{I}(G)$. Then $(1,1) \in$ $\mathcal{I}(G)$.

4. Graphs whose minimum rank is two and whose positive semidefinite minimum rank is $\mathbf{k}$. We provide the following theorem and proof preliminary to a larger result.

THEOREM 4.1. Given a connected graph $G$, the following statements are equivalent:

1. $\operatorname{mr}(G)=\mathrm{mr}_{+}(G)=2$.

2. $G^{c}$ is nonempty and can be expressed as the union of complete bipartite graphs.

3. $G$ is $\left(P_{4}\right.$, dart, $\left.\ltimes, K_{3,3,3}\right)$-free and $\alpha(G)=2$.

Proof.

$1 \Rightarrow 3$

By Theorem $3.2, \operatorname{mr}(G)=2 \Rightarrow G$ is $\left(P_{4}\right.$, dart, $\left.\ltimes, K_{3,3,3}\right)$-free. By Theorem 3.4, we know $\alpha(G) \leq \operatorname{mr}_{+}(G)=2$. If $\alpha(G) \leq 1$, then $G$ is a complete graph and $\operatorname{mr}(G)=1$, a contradiction. Therefore, $\alpha(G)=2$.

$3 \Rightarrow 2$

It follows from Theorem 3.3 that $G^{c}$ is the union of complete bipartite graphs and since $\omega\left(G^{c}\right)=\alpha(G)=2, G^{c}$ is nonempty. 
$2 \Rightarrow 1$

By Theorem 3.3, $\mathrm{mr}_{+}(G) \leq 2$. If $\operatorname{mr}(G)=1, G$ is a complete graph and $G^{c}$ is empty, a contradiction. Therefore, $2 \leq \operatorname{mr}(G) \leq \mathrm{mr}_{+}(G) \leq 2$.

Lemma 4.2. Let $k \geq 3$. Then it is possible to construct sets of vectors $\left\{u_{1}, u_{2}, \ldots, u_{k}\right\},\left\{v_{1}, v_{2}, \ldots, v_{k}\right\},\left\{x_{1}, y_{1}\right\},\left\{x_{2}, y_{2}\right\}, \ldots,\left\{x_{t}, y_{t}\right\}$ in $\mathbb{R}^{k}$ such that all vectors within a given set are pairwise orthogonal and such that any pair of vectors from distinct sets is not orthogonal.

Proof. Consider the following vectors:

$$
\begin{array}{cc}
u_{1}=[1,0,0,0, \ldots, 0,0,0] & u_{2}=[0,1,0,0, \ldots, 0,0,0] \\
\ldots & \ldots \\
u_{k-1}=[0,0,0,0, \ldots, 0,1,0] & u_{k}=[0,0,0,0, \ldots, 0,0,1] \\
v_{1}=\left[1-\frac{k}{2}, 1,1, \ldots, 1,1\right] & v_{2}=\left[1,1-\frac{k}{2}, 1, \ldots, 1,1\right] \\
\ldots & \ldots \\
v_{k-1}=\left[1,1,1, \ldots, 1-\frac{k}{2}, 1\right] & v_{k}=\left[1,1,1, \ldots, 1,1-\frac{k}{2}\right] .
\end{array}
$$

For odd k:

$$
\begin{array}{cc}
x_{1}=\left[\cos \theta_{1}, \sin \theta_{1}, \cos \theta_{1}, \ldots, \sin \theta_{1}, 1\right] & y_{1}=\left[\cos \theta_{1}, \sin \theta_{1}, \cos \theta_{1}, \ldots, \sin \theta_{1}, \frac{1-k}{2}\right] \\
x_{2}=\left[\cos \theta_{2}, \sin \theta_{2}, \cos \theta_{2}, \ldots, \sin \theta_{2}, 1\right] & y_{2}=\left[\cos \theta_{2}, \sin \theta_{2}, \cos \theta_{2}, \ldots, \sin \theta_{2}, \frac{1-k}{2}\right] \\
\vdots & \vdots \\
x_{t}=\left[\cos \theta_{t}, \sin \theta_{t}, \cos \theta_{t}, \ldots, \sin \theta_{t}, 1\right] & y_{t}=\left[\cos \theta_{t}, \sin \theta_{t}, \cos \theta_{t}, \ldots, \sin \theta_{t}, \frac{1-k}{2}\right]
\end{array}
$$

For even k:

$$
\begin{array}{cc}
x_{1}=\left[\cos \theta_{1}, \sin \theta_{1}, \cos \theta_{1}, \ldots, \sin \theta_{1}\right] & y_{1}=\left[-\sin \theta_{1}, \cos \theta_{1},-\sin \theta_{1}, \ldots, \cos \theta_{1}\right] \\
x_{2}=\left[\cos \theta_{2}, \sin \theta_{2}, \cos \theta_{2}, \ldots, \sin \theta_{2}\right] & y_{2}=\left[-\sin \theta_{2}, \cos \theta_{2},-\sin \theta_{2}, \ldots, \cos \theta_{2}\right] \\
\vdots & \vdots \\
x_{t}=\left[\cos \theta_{t}, \sin \theta_{t}, \cos \theta_{t}, \ldots, \sin \theta_{t}\right] & y_{t}=\left[-\sin \theta_{t}, \cos \theta_{t},-\sin \theta_{t}, \ldots, \cos \theta_{t}\right] .
\end{array}
$$

We choose $\theta_{i}$ such that $0<\theta_{1}<\theta_{2}<\cdots<\theta_{t}<\frac{\pi}{2}$, and $\theta_{i} \neq \arccos \left(\frac{k-2}{\sqrt{2}(k-1)}\right)+\frac{\pi}{4}$ for all $i$.

I. All vectors within a set are pairwise orthogonal.

The $u$ set has only one non-zero term in each vector and none of the other vectors 
have a non-zero term in the same location so they must be orthogonal to each other,

$$
\begin{aligned}
v_{r} \cdot v_{s} & =1+1+\cdots+1-\frac{k}{2}+\cdots+1-\frac{k}{2}+\cdots+1 \\
& =\sum_{j=1}^{k} 1-\frac{k}{2}-\frac{k}{2}=k-k=0 .
\end{aligned}
$$

For odd k:

$$
\begin{aligned}
x_{r} \cdot y_{r} & =\left(\cos ^{2} \theta_{r}+\sin ^{2} \theta_{r}\right)+\left(\cos ^{2} \theta_{r}+\sin ^{2} \theta_{r}\right)+\cdots+\frac{1-k}{2} \\
& =1+1+\cdots+1+\frac{1-k}{2}=\frac{k-1}{2}+\frac{1-k}{2}=0 .
\end{aligned}
$$

For even k:

$$
\begin{aligned}
x_{r} \cdot y_{r} & =-\cos \theta_{r} \sin \theta_{r}+\cos \theta_{r} \sin \theta_{r}-\cdots-\cos \theta_{r} \sin \theta_{r}+\cos \theta_{r} \sin \theta_{r} \\
& =\frac{k}{2}\left(\cos \theta_{r} \sin \theta_{r}-\cos \theta_{r} \sin \theta_{r}\right)=0 .
\end{aligned}
$$

II. Vectors from distinct sets are not orthogonal.

Any vector in the $u$ set has only one nonzero term, and no vectors in the other sets have zero terms so the $u$ vectors cannot be orthogonal to a vector in a different set.

\section{For odd k:}

$$
\begin{array}{rlrl}
v_{r} \cdot x_{s} & =\cos \theta_{s}+\sin \theta_{s}+\cdots+\left(1-\frac{k}{2}\right) \cos \theta_{s}+\cdots+1 & & \text { for odd } r \neq k \\
(1) & =\left(\frac{k-1}{2}\right)\left(\cos \theta_{s}+\sin \theta_{s}\right)-\frac{k}{2} \cos \theta_{s}+1 & \text { for } 0<\theta_{s}<\frac{\pi}{2} \\
(2) & =\left(\frac{k-1}{2}\right) \sin \theta_{s}-\frac{1}{2} \cos \theta_{s}+1>0 & & \text { for odd } r \neq k \\
v_{r} \cdot y_{s} & =\cos \theta_{s}+\sin \theta_{s}+\cdots+\left(1-\frac{k}{2}\right) \cos \theta_{s}+\cdots+\frac{1-k}{2} & \\
(3) & =\left(\frac{k-1}{2}\right)\left(\cos \theta_{s}+\sin \theta_{s}\right)-\frac{k}{2} \cos \theta_{s}-\frac{k-1}{2} & & \text { for } 0<\theta_{s}<\frac{\pi}{2} . \\
(4) & =-\left(\frac{k-1}{2}\right)\left(1-\sin \theta_{s}\right)-\frac{1}{2} \cos \theta_{s}<0 &
\end{array}
$$


For even $r \neq k$ change the $-\frac{k}{2} \cos$ to a $-\frac{k}{2} \sin$ in (1) and (3) and you will get an inequality similar to (2) and (4) with the cos's and sin's switched,

$$
\begin{aligned}
& v_{k} \cdot x_{s}=\cos \theta_{s}+\sin \theta_{s}+\cos \theta_{s}+\cdots+\left(1-\frac{k}{2}\right) \\
& =\left(\frac{k-1}{2}\right)\left(\cos \theta_{s}+\sin \theta_{s}\right)-\frac{k-2}{2} \\
& =\frac{\sqrt{2}(k-1)}{2} \cos \left(\theta_{s}-\frac{\pi}{4}\right)-\frac{k-2}{2} \neq 0 \\
& \text { by the choice of } \theta_{i} \text { 's } \\
& v_{k} \cdot y_{s}=\cos \theta_{s}+\sin \theta_{s}+\cos \theta_{s}+\cdots+\left(1-\frac{k}{2}\right)\left(\frac{1-k}{2}\right) \\
& =\left(\frac{k-1}{2}\right)\left(\cos \theta_{s}+\sin \theta_{s}+\frac{k-2}{2}\right)>0 \\
& x_{r} \cdot x_{s}=\cos \theta_{r} \cos \theta_{s}+\sin \theta_{r} \sin \theta_{s}+\cdots+1>0 \\
& x_{r} \cdot y_{s}=\cos \theta_{r} \cos \theta_{s}+\sin \theta_{r} \sin \theta_{s}+\cdots+\frac{1-k}{2} \\
& =\left(\frac{k-1}{2}\right)\left(\cos \theta_{r} \cos \theta_{s}+\sin \theta_{r} \sin \theta_{s}-1\right) \\
& =\left(\frac{k-1}{2}\right)\left(\cos \left(\theta_{r}-\theta_{s}\right)-1\right) \neq 0 \\
& y_{r} \cdot y_{s}=\cos \theta_{r} \cos \theta_{s}+\sin \theta_{r} \sin \theta_{s}+\cdots+\frac{(1-k)^{2}}{4}>0 \quad \text { for } 0<\theta_{r}, \theta_{s}<\frac{\pi}{2} \text {. }
\end{aligned}
$$

\section{For even k:}

$$
\begin{aligned}
v_{r} \cdot x_{s} & =\cos \theta_{s}+\sin \theta_{s}+\cdots+\left(1-\frac{k}{2}\right) \cos \theta_{s}+\cdots+\sin \theta_{s} & & \text { for odd } r \\
& =\frac{k}{2}\left(\cos \theta_{s}+\sin \theta_{s}\right)-\frac{k}{2} \cos \theta_{s}=\frac{k}{2} \sin \theta_{s}>0 & & \text { for } 0<\theta_{s}<\frac{\pi}{2} \\
v_{r} \cdot y_{s} & =-\sin \theta_{s}+\cos \theta_{s}-\cdots-\left(1-\frac{k}{2}\right) \sin \theta_{s}+\cdots+\cos \theta_{s} & & \text { for odd } r \\
& =\frac{k}{2}\left(\cos \theta_{s}-\sin \theta_{s}\right)+\frac{k}{2} \sin \theta_{s}=\frac{k}{2} \cos \theta_{s}>0 & & \text { for } 0<\theta_{s}<\frac{\pi}{2} .
\end{aligned}
$$


For even $r$ change the equations in the same manner as the odd $k$ case,

$$
\begin{aligned}
x_{r} \cdot x_{s} & =\cos \theta_{r} \cos \theta_{s}+\sin \theta_{r} \sin \theta_{s}+\cdots+\sin \theta_{r} \sin \theta_{s}>0 & & \text { for } 0<\theta_{r}, \theta_{s}<\frac{\pi}{2} \\
x_{r} \cdot y_{s} & =-\cos \theta_{r} \sin \theta_{s}+\cos \theta_{s} \sin \theta_{r}-\cdots+\cos \theta_{s} \sin \theta_{r} & & \\
& =\frac{k}{2}\left(\sin \theta_{r} \cos \theta_{s}-\cos \theta_{r} \sin \theta_{s}\right)=\frac{k}{2} \sin \left(\theta_{r}-\theta_{s}\right) \neq 0 & & \text { for } \theta_{r} \neq \theta_{s} \\
y_{r} \cdot y_{s} & =\sin \theta_{r} \sin \theta_{s}+\cos \theta_{r} \cos \theta_{s}+\cdots+\cos \theta_{r} \cos \theta_{s}>0 & & \text { for } 0<\theta_{r}, \theta_{s}<\frac{\pi}{2} .
\end{aligned}
$$

THEOREM 4.3. Given a connected graph $G$ and any integer $k \geq 2$, the following statements are equivalent:

1. $m r(G)=2$ and $\operatorname{mr}_{+}(G)=k$.

2. $G^{c}=K_{k} \cup K_{\ell} \cup$ complete bipartite graphs, where $0 \leq \ell \leq k$. If $\ell=0$ there is at least one complete bipartite graph in the union.

3. $G$ is $\left(P_{4}\right.$, dart, $\left.\ltimes, K_{3,3,3}\right)$-free and $\alpha(G)=k$.

Proof. We have shown that this theorem is true for $k=2$. Assume that it is true for all integers less than $k$ and $\geq 2$.

$1 \Rightarrow 3$

By Theorem $3.2, \operatorname{mr}(G)=2 \Rightarrow G$ is $\left(P_{4}\right.$, dart, $\left.\ltimes, K_{3,3,3},\right)$-free. Also, since $\alpha(G)$ is a lower bound on $\mathrm{mr}_{+}(G)$, we know $\alpha(G) \leq \mathrm{mr}_{+}(G)=k$. If $\alpha(G)=r<k$, by the inductive hypothesis $\mathrm{mr}_{+}(G)=r<k$, a contradiction. Therefore $\alpha(G) \geq k$, hence we have $\alpha(G)=k$.

$3 \Rightarrow 2$

By Theorem 3.2, $G^{c}$ can be expressed as the union of at most two complete graphs and of complete bipartite graphs. Since $\alpha(G)=k$, the complement has at least one complete graph of size $k$ giving the component $K_{k}$, and no larger complete graphs giving $\ell \leq k$ for the second component $K_{\ell}$. If $\ell=0$ and the third set is empty then $G=K_{k}^{c}$, a contradiction.

$2 \Rightarrow 1$

Suppose $G^{c}=K_{k} \cup K_{\ell} \cup K_{p_{1}, q_{1}} \cup \cdots \cup K_{p_{t}, q_{t}}$. Then by Theorem 3.2 we have $\operatorname{mr}(G)=2$. Also, since $\mathrm{G}$ contains $K_{k}^{c}$, we have $\alpha(G) \geq k$ which implies $\operatorname{mr}_{+}(G) \geq k$. We also know that any matrix $M \in \mathcal{S}(G)$ has the form 


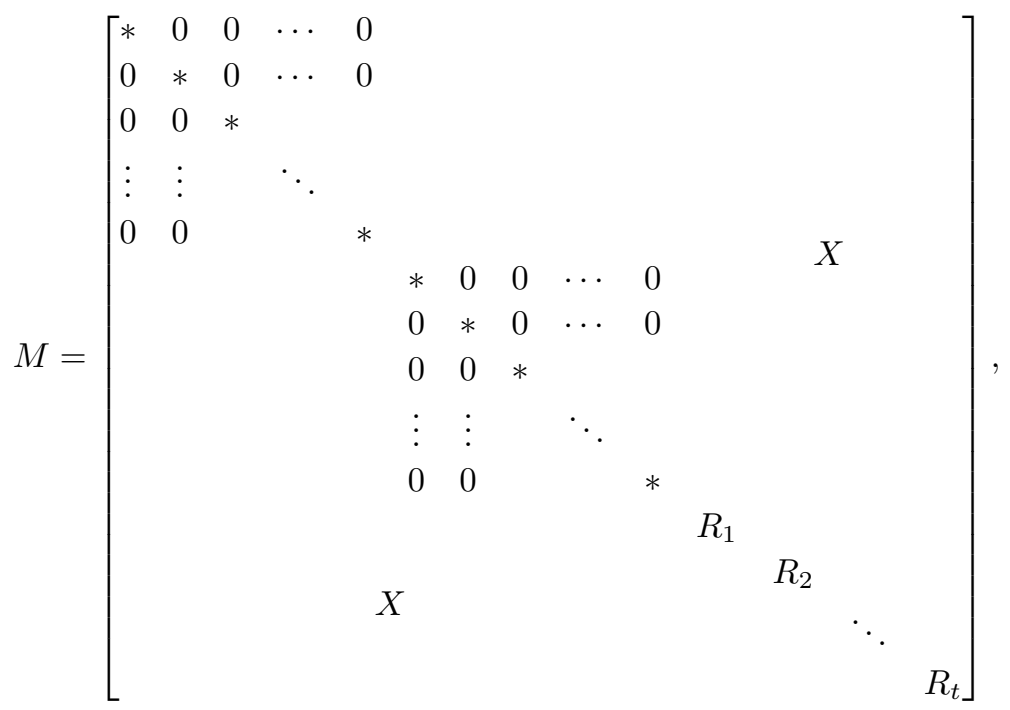

where the first two blocks are of size $k$ and $\ell$, where $R_{i}=\left[\begin{array}{cc}S_{i} & 0 \\ 0 & T_{i}\end{array}\right]$ for $S_{i} \in \mathcal{S}\left(K_{p_{i}}\right)$ and $T_{i} \in \mathcal{S}\left(K_{q_{i}}\right)$, and where $X$ denotes the obvious nonzero structure of the matrix.

Assuming $G$ has $n$ vertices, $M$ is an $n \times n$ matrix. Suppose there exists a $k \times n$ matrix $B$, such that $M=B^{T} B \in \mathcal{S}(G)$. Since M is a Gram matrix, it's positive semidefinite, and thus we could conclude $\operatorname{mr}_{+}(G) \leq k$. Hence, $k \leq \operatorname{mr}_{+}(G) \leq k$ so we know $\mathrm{mr}_{+}(G)=k$. We will show it is possible to build such a matrix $M=B^{T} B \in$ $\mathcal{S}(G)$ using the vectors constructed in Lemma 4.2 .

We define

$$
\begin{aligned}
& U=\left[\begin{array}{llll}
u_{1}^{T} & u_{2}^{T} & \cdots & u_{k}^{T}
\end{array}\right], \\
& V=\left[\begin{array}{llll}
v_{1}^{T} & v_{2}^{T} & \cdots & v_{\ell}^{T}
\end{array}\right], \\
& W_{i}=\left[\begin{array}{ll}
x_{i}^{T} \cdots x_{i}^{T} & y_{i}^{T} \cdots y_{i}^{T}
\end{array}\right] \text { so that } R_{i}=W_{i}^{T} W_{i} \in \mathcal{S}\left(K_{p_{i}} \cup K_{q_{i}}\right) \text {, } \\
& B=\left[\begin{array}{llllll}
U & V & W_{1} & W_{2} & \cdots & W_{t}
\end{array}\right] \text {. }
\end{aligned}
$$

Then let $M=B^{T} B$. Because of the orthogonality conditions in Lemma 4.2, $M \in \mathcal{S}(G)$ with $\operatorname{rank}(M) \leq k$.

Corollary 4.4. Let $G$ be a connected graph. If $\operatorname{mr}(G)=2$, then $\operatorname{mr}_{+}(G)=$ $\alpha(G)$.

Proof. Since $\operatorname{mr}_{+}(G) \geq \operatorname{mr}(G)=2$, by Theorem $4.3, \mathrm{mr}_{+}(G)=\alpha(G)$. 
A more general result than Corollary 4.4 was found earlier by Hein van der Holst, Raphael Loewy, and Barrett, but has not been published. We include that result and proof with their permission.

ThEOREM 4.5. For a decomposable graph $G$,

$$
\mathrm{mr}_{+}(G)+\iota(G)=\alpha(G),
$$

where $\iota(G)$ is the number of isolated vertices in $G$.

The proof uses the following lemmas, which are Corollary 2.7 and Proposition 2.6 in $[8]$.

Lemma 4.6. For any graph $G, \mathrm{mr}_{+}\left(G \vee K_{1}\right)=\mathrm{mr}_{+}(G)+\iota(G)$.

LEMmA 4.7. For any graphs $G$ and $H$,

$$
\operatorname{mr}_{+}(G \vee H)=\max \left\{\mathrm{mr}_{+}\left(G \vee K_{1}\right), \operatorname{mr}_{+}\left(H \vee K_{1}\right)\right\}
$$

Combining the two lemmas we obtain the following:

Lemma 4.8. For any graphs $G$ and $H$,

$$
\operatorname{mr}_{+}(G \vee H)=\max \left\{\operatorname{mr}_{+}(G)+\iota(G), \operatorname{mr}_{+}(H)+\iota(H)\right\} .
$$

We can now prove Theorem 4.5 by induction on the number of vertices of $G$.

Proof. For $G=K_{1}: \mathrm{mr}_{+}\left(K_{1}\right)+\iota\left(K_{1}\right)=0+1=\alpha\left(K_{1}\right)$.

We verify that if the equality holds for $G$ and $H$, then it holds for $G \cup H$ and $G \vee H$.

Unions: $\mathrm{mr}_{+}(G \cup H)+\iota(G \cup H)=\mathrm{mr}_{+}(G)+\mathrm{mr}_{+}(H)+\iota(G)+\iota(H)=\alpha(G)+$ $\alpha(H)=\alpha(G \cup H)$.

Joins: By Lemma 4.8, $\operatorname{mr}_{+}(G \vee H)=\max \left\{\operatorname{mr}_{+}(G)+\iota(G), \operatorname{mr}_{+}(H)+\iota(H)\right\}=$ $\max \{\alpha(G), \alpha(H)\}=\alpha(G \vee H)$.

Since every minimum rank 2 graph is decomposable, Corollary 4.4 is an immediate consequence of Theorem 4.5. Some results in the next section depend only on Corollary 4.4 making this second short approach to the corollary preferable. But it is more transparent to view the inverse eigenvalue problem in the final section in terms of Theorem 4.3, so we have retained its lengthy proof. Moreover, the proof of $2 \Rightarrow 1$ also gives a construction of a positive semidefinite matrix of rank $k$ for a graph $G$ with $\operatorname{mr}(G)=2$ and $\mathrm{mr}_{+}(G)=k$.

We have reduced the problem of finding the minimum positive semidefinite rank of graphs with minimum rank two to finding the independence number. While this 
is difficult for graphs in general, the proof of Theorem 4.5 shows that it can be done easily for decomposable graphs. See [7] for further details on finding the independence number of a decomposable graph.

The results presented thus far solve the inverse inertia problem for graphs whose minimum rank is two. Let $G$ be a graph on $n$ vertices whose minimum rank is two. By Corollary 3.8, $(1,1) \in \mathcal{I}(G)$. Using the fact that $\mathrm{mr}_{+}(G)=\alpha(G)$ and the Northeast Lemma, we conclude that the inertia set of $G$ includes every possible point except the points along the $\mathrm{x}$-axis whose $\mathrm{x}$ coordinate is less than $\alpha(G)$ and the points along the $\mathrm{y}$-axis whose y coordinate is less than $\alpha(G)$. The following example is $K S_{2,4}$ (see [5] for this notation). It's known that $\operatorname{mr}\left(K S_{2,4}\right)=2$ and $\alpha\left(K S_{2,4}\right)=4$. We show the inertia set by plotting the points in $\mathbb{R}^{2}$.
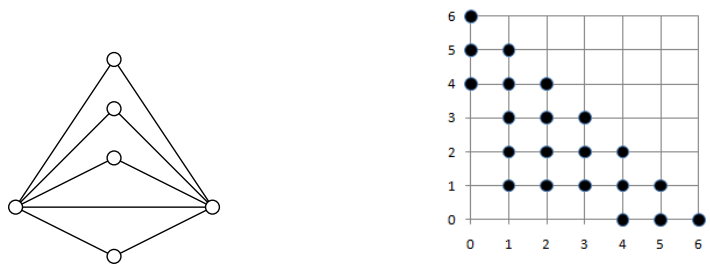

Now that we have shown that $\operatorname{mr}_{+}(G)=\alpha(G)$ provided $\operatorname{mr}(G)=2$, we ask, how are $\operatorname{mr}_{+}(G)$ and $\alpha(G)$ related when $\operatorname{mr}(G) \neq 2$ ? Assuming no restrictions are placed on $\mathrm{mr}_{+}(G)$ and $\alpha(G)$, we note that the difference $\mathrm{mr}_{+}(G)-\alpha(G)$ may be arbitrarily large. Consider $P_{n}$. Then $\mathrm{mr}_{+}\left(P_{n}\right)-\alpha\left(P_{n}\right)=n-1-\lfloor n / 2\rfloor \rightarrow \infty$ as $n \rightarrow \infty$. For $\operatorname{mr}(G) \neq 2$, the question concerning the relationship of $\mathrm{mr}_{+}(G)$ and $\alpha(G)$ for a fixed $\alpha(G)$ remains open.

5. Nil, neutral, and nonzero vertices of graphs whose minimum rank is two. Another interesting generalization of the Minimum Rank Problem asks, given a graph $G$ what structures are possible for matrices $A \in \mathcal{S}(G)$ with $\operatorname{rank} A=\operatorname{mr}(G)$ ? More concisely, what are the structures of matrices in $\mathscr{M} \mathscr{R}(G)$ ? A first step towards classifying $\mathscr{M} \mathscr{R}(G)$ is determining which diagonal entries, if any, are required to be zero or nonzero (see Definition 2.14). There are cases when no diagonal entry is required to be zero. For example, if a connected graph $G$ has $\operatorname{mr}_{+}(G)=\operatorname{mr}(G)$ (note: this occurs for all graphs with a trapezoidal inertia set), then there exists a positive semidefinite matrix in $\mathscr{M} \mathscr{R}(G)$. If a diagonal entry was zero, the entire row and column of the matrix containing this entry would be zero in order for the matrix to be positive semidefinite. This contradicts $G$ being a connected graph. Thus, $\mathrm{mr}_{+}(G) \neq \operatorname{mr}(G)$ is a necessary condition for $G$ to have a nil vertex. We note however that this is not a sufficient condition. For example, using techniques found in [10], we know that for the graph $G$ below, $\operatorname{mr}(G)=6, \operatorname{mr}_{+}(G)=7$ and $G$ has no nil vertices. 


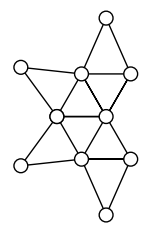

The following theorems characterize nil, neutral, and nonzero vertices of graphs whose minimum rank is two. For results of a more general nature about nil vertices see $[2]$.

THEOREM 5.1. Let $G$ be a connected graph and assume $\operatorname{mr}(G)=2$ and $\alpha(G)>2$. Let $S$ be an independent set of size $k>2$. Then every vertex in $S$ is a nil vertex.

Proof. Let $M \in \mathscr{M} \mathscr{R}(G)$, and assume $|G|=n$. Since $M$ is real symmetric, it can be expressed as the sum of rank 1 matrices, $M=\lambda_{1} q_{1} q_{1}^{T}+\lambda_{2} q_{2} q_{2}^{T}+\cdots+\lambda_{n} q_{n} q_{n}^{T}$, where the $\lambda_{i}$ are the eigenvalues of $M$ and each $q_{i} \in \mathbb{R}^{n}$. Since $\operatorname{rank}(M)=\operatorname{mr}(G)=2$, $M$ has 2 nonzero eigenvalues and the eigenvalue 0 with multiplicity $n-2$. By Corollary $4.4, \mathrm{mr}_{+}(G)=\alpha(G)>2$. Thus the two nonzero eigenvalues must be of opposite sign. Say $\lambda_{1}>0$ and $\lambda_{2}<0$. So $M$ reduces to the sum of two rank 1 matrices, and we can write $M=\sqrt{\lambda_{1}} q_{1}\left(\sqrt{\lambda_{1}} q_{1}\right)^{T}-\sqrt{-\lambda_{2}} q_{2}\left(\sqrt{-\lambda_{2}} q_{2}\right)^{T}$. Thus we can write $M$ as the difference of two rank 1 matrices $A=a a^{T}$ and $B=b b^{T}$.

Label the vertices of the independent set $S$ as $1, \ldots, k$. Let $*$ represent an entry that may be zero or nonzero. Then

$$
\begin{aligned}
& M=A-B=\left[\begin{array}{ccccccc}
d_{1} & 0 & \cdots & 0 & * & \cdots & * \\
0 & d_{2} & \ddots & \vdots & \vdots & & \vdots \\
\vdots & \ddots & \ddots & 0 & \vdots & & \\
0 & \ldots & 0 & d_{k} & * & & \\
* & \ldots & \ldots & * & \ddots & \ddots & \vdots \\
\vdots & & & & \ddots & \ddots & * \\
* & \ldots & & & \ldots & * & d_{n}
\end{array}\right] \\
& =\left[\begin{array}{cccccc}
a_{1}^{2} & a_{1} a_{2} & \cdots & a_{1} a_{k} & \cdots & a_{1} a_{n} \\
a_{2} a_{1} & a_{2}^{2} & & a_{2} a_{k} & & a_{2} a_{n} \\
\vdots & & \ddots & \vdots & & \vdots \\
a_{k} a_{1} & a_{k} a_{2} & \cdots & a_{k}^{2} & & a_{i} a_{k} \\
\vdots & & & & \ddots & \vdots \\
a_{n} a_{1} & a_{n} a_{2} & \cdots & a_{n} a_{k} & \cdots & a_{n}^{2}
\end{array}\right]-\left[\begin{array}{cccccc}
b_{1}^{2} & b_{1} b_{2} & \cdots & b_{1} b_{k} & \cdots & b_{1} b_{n} \\
b_{2} b_{1} & b_{2}^{2} & & b_{2} b_{k} & & b_{2} b_{n} \\
\vdots & & \ddots & \vdots & & \vdots \\
b_{k} b_{1} & b_{k} b_{2} & \cdots & b_{k}^{2} & \cdots & b_{k} b_{n} \\
\vdots & & & \vdots & \ddots & \vdots \\
b_{n} b_{1} & b_{n} b_{2} & \cdots & b_{n} b_{k} & \cdots & b_{n}^{2}
\end{array}\right] .
\end{aligned}
$$


Let $r, s, t \in S$ be distinct. Since $m_{r s}, m_{r t}, m_{s t}=0$, corresponding entries in $A$ and $B$ are equal. Thus

$$
\begin{aligned}
& a_{r} a_{s}=b_{r} b_{s}, \\
& a_{r} a_{t}=b_{r} b_{t}, \\
& a_{s} a_{t}=b_{s} b_{t} .
\end{aligned}
$$

I. $a_{r} a_{s} a_{t} b_{r} b_{s} b_{t}=0$. Without loss of generality suppose $a_{r}=0$. Then by (5.1) and (5.2)

$$
b_{r} b_{s}=a_{r} a_{s}=0=a_{r} a_{t}=b_{r} b_{t},
$$

from which we have the following two possibilities:

i. $b_{r}=0$ : Then $r$ is an isolated vertex, which is impossible.

ii. $b_{s}=b_{t}=0$ : Then

$$
a_{s} a_{t}=b_{s} b_{t}=0
$$

so either $a_{s}=0$ or $a_{t}=0$. Then either $s$ or $t$ is an isolated vertex, and we have the same contradiction as before.

II. We may now suppose that $a_{r} a_{s} a_{t} b_{r} b_{s} b_{t} \neq 0$. Then (5.1), (5.2), and (5.3) give

$$
\begin{aligned}
& \frac{a_{r}}{b_{r}}=\frac{b_{s}}{a_{s}}, \\
& \frac{a_{r}}{b_{r}}=\frac{b_{t}}{a_{t}}, \\
& \frac{a_{s}}{b_{s}}=\frac{b_{t}}{a_{t}},
\end{aligned}
$$

and those equations together give

$$
\frac{a_{s}}{b_{s}}=\frac{b_{t}}{a_{t}}=\frac{a_{r}}{b_{r}}=\frac{b_{s}}{a_{s}} \Rightarrow a_{s}^{2}=b_{s}^{2} .
$$

Hence, $d_{s}=a_{s}^{2}-b_{s}^{2}=0$. By symmetry, $d_{i}=0$ for $i=1, \ldots, k$. $\square$

Example: We will use the above theorem to show that every vertex of $K_{m, n}$ with $m, n \geq 3$ is a nil vertex. Consider $K_{m, n}$ with $m, n \geq 3$. It is known that $\operatorname{mr}\left(K_{m, n}\right)=2$. 
By Corollary 4.4, $\operatorname{mr}_{+}\left(K_{m, n}\right)=\alpha\left(K_{m, n}\right)=\max \{m, n\} \geq 3$. The two independent sets of size $m$ and $n$ contain every vertex of $K_{m, n}$. By Theorem 5.1, every vertex is a nil vertex.

The following theorems will show that the nil vertices of a graph whose minimum rank is two can only be found in independent sets of size 3 or more.

THEOREM 5.2. If $H$ is an induced subgraph of a graph $G$ with $\operatorname{mr}(H)=\operatorname{mr}(G)$, then if $v$ is a nil (nonzero) vertex in $H v$ is also a nil (nonzero) vertex in $G$.

Proof. Let $G, H$, and $v$ be as described above. Let $A \in \mathscr{M} \mathscr{R}(G)$. Let $B$ be the principal submatrix of $A$ corresponding to $H$. Since $\operatorname{rank} A=\operatorname{mr}(G)=\operatorname{mr}(H)$, $\operatorname{rank} B=\operatorname{mr}(H)$. Thus $B \in \mathscr{M} \mathscr{R}(H)$, and so the diagonal entry of $A$ corresponding to $v$ is zero (nonzero). Thus $v$ is a nil (nonzero) vertex in $G$.

Lemma 5.3. Let $G$ be a paw $\left(\bigcap_{0}-0\right)$. The non-dominating vertices of $G$ are nonzero.

Proof. Let $G$ be the paw and let $A \in \mathcal{S}(G)$ have rank 2 .

$$
A=\left[\begin{array}{cccc}
d_{1} & a & b & 0 \\
a & d_{2} & c & 0 \\
b & c & d_{3} & e \\
0 & 0 & e & d_{4}
\end{array}\right]
$$

Suppose that at least one of $d_{1}, d_{2}, d_{4}$ is equal to zero.

If $d_{4} \neq 0$, then at least one of $d_{1}$ and $d_{2}$ must be zero. Then the matrix $A(3)$ below has full rank.

$$
A(3)=\left[\begin{array}{ccc}
d_{1} & a & 0 \\
a & d_{2} & 0 \\
0 & 0 & d_{4}
\end{array}\right]
$$

If $d_{4}=0$, then the matrix formed by deleting row 1 and column 2 from $A$ has full rank.

In each case we contradict the fact that $A$ has rank 2. Thus none of $d_{1}, d_{2}, d_{4}$ may be zero.

LEMma 5.4. The dominating vertex of $K_{3,3} \vee K_{1}=\left(K_{3} \cup K_{3} \cup K_{1}\right)^{c}=K_{3,3,1}$ is nonzero.

Proof. Since $\operatorname{mr}\left(K_{3,3,1}\right)=2$, by Theorem 5.1 the vertices that constitute the independent sets of size 3 are nil vertices. Hence, any matrix $M \in \mathcal{S}\left(K_{3,3,1}\right)$ has the form 


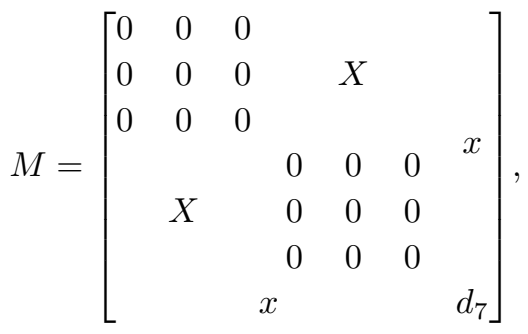

where $X$ and $x$ denote the obvious nonzero structure of the matrix.

Consider the $3 \times 3$ submatrix of $M, M[\{1,4,7\}]=\left[\begin{array}{ccc}0 & a & b \\ a & 0 & c \\ b & c & d_{7}\end{array}\right], a b c \neq 0$. Suppose $d_{7}=0$. Then $M[\{1,4,7\}]$ would be an invertible submatrix of $M$, contradicting rank $M=2$. Therefore, $d_{7} \neq 0$, and hence the corresponding vertex is nonzero.

Corollary 5.5. Let $G$ be a graph with $\operatorname{mr}(G)=2$. If $G$ has an induced paw or an induced $K_{3,3,1}$, then the non-dominating vertices of the former and the dominating vertex of the latter are nonzero.

TheOREM 5.6. Let $G$ be a connected graph with $\operatorname{mr}(G)=2$ and $v$ be a vertex of G. Then

- $v$ is a nonzero vertex if and only if $v$ is either a non-dominating vertex of an induced paw of $G$ or else is the dominating vertex of an induced $K_{3,3,1}$.

- $v$ is a nil vertex if and only if $v$ is in an independent set of size three or greater.

- $v$ is a neutral vertex if and only if it does not meet either of the previous two conditions.

Proof. The reverse implication of each of the first two statements follows from Theorem 5.1 and Corollary 5.5. It now suffices to show the reverse implication of the third statement.

Let $v$ be a vertex not meeting the stated conditions to be a nil or nonzero vertex. In the case that $K_{3,3}$ is induced in $G$, every vertex not in the two independent sets of size three or greater is a dominating vertex in an induced $K_{3,3,1}$ and so there are no such $v$. In this case Theorem 5.6 is satisfied and $G$ has only nil and nonzero vertices.

We now need only consider cases where $G$ has at most one independent set of size three or greater and may without loss of generality let

$$
G=\left(K_{k} \cup K_{m_{1}, n_{1}} \cup \cdots \cup K_{m_{r}, n_{r}} \cup s K_{2} \cup t K_{1}\right)^{c},
$$


where $s, t \geq 0, k \in\{0,3,4, \ldots\}$, and none of the $K_{m_{i}, n_{i}}$ are $K_{2}$ 's or $K_{1}$ 's. Note $G$ does not have $K_{3,3,1}$ as an induced subgraph. We identify all vertices that are nondominating vertices in an induced paw. Since $G^{c}$ is the union of at least two graphs (else $G$ would not be connected or $\operatorname{mr}(G) \neq 2$ ), if $K_{2,1}$ is an induced subgraph of $G^{c}$, the paw $=\left(K_{2,1} \cup K_{1}\right)^{c}$ is an induced subgraph of $G$. Since the non-dominating vertices of the paw correspond to the $K_{2,1}$, all vertices of $G$ corresponding to the $K_{m_{i}, n_{i}}$ are non-dominating vertices of induced paws. The remaining vertices that are not in an independent set of size three or greater correspond to the $K_{1}$ 's and $K_{2}$ 's. The $K_{1}$ 's are dominating vertices in $G$, and hence cannot be non-dominating vertices of an induced paw. The vertices of the $K_{2}$ 's will have the same neighborhood and be nonadjacent in $G$, and hence cannot be two non-dominating vertices of an induced paw. Thus all vertices of $G$ corresponding to the non-dominating vertices of induced paws are exactly those vertices corresponding to the $K_{m_{i}, n_{i}}$. Therefore $v$ corresponds to vertices of the $K_{1}$ 's and $K_{2}$ 's.

Given a matrix in $\mathscr{M} \mathscr{R}(G)$, we show the vertices corresponding to the $K_{1}$ 's and $K_{2}$ 's can have either zero or nonzero corresponding diagonal entries. The methods used here are used extensively and to greater effect in the succeeding section and thus may serve as a familiarization to the reader. For the moment we simply use the following vectors to construct matrices in $\mathscr{M} \mathscr{R}(G)$. Let

$$
\begin{array}{ll}
x=[a, a], & y=[a,-a], \\
u_{i}=\left[\cos \theta_{i}, \sin \theta_{i}\right], & v_{i}=\left[\sin \theta_{i}, \cos \theta_{i}\right],
\end{array}
$$

where $0<\theta_{1}<\theta_{2}<\cdots<\theta_{r+s+t}<\frac{\pi}{4}$. Define $X$ to be the $2 \times 2$ matrix with the vector $x$ in each row; $U_{i}$ to be the $\left(m_{i}+n_{i}\right) \times 2$ matrix with the vector $u_{i}$ in the first $m_{i}$ rows, and $v_{i}$ in the last $n_{i}$ rows for each $1 \leq i \leq r$; and $W_{i}$ to be the $2 \times 2$ matrix with the vector $u_{i}$ in the first row and $v_{i}$ in the second row for each $r+1 \leq i \leq r+s$.

Suppose $k=0$ and $s, t \geq 1$. We now construct a matrix $B \in \mathscr{M} \mathscr{R}(G)$ with zero diagonal entries corresponding to one $K_{2}$ and one $K_{1}$ and nonzero diagonal entries corresponding to all other vertices. Let

$$
A=\left[\begin{array}{lllllllllll}
U_{1}^{T} & \cdots & U_{r}^{T} & X^{T} & W_{r+2}^{T} & \cdots & W_{r+s}^{T} & y^{T} & u_{r+s+2}^{T} & \cdots & u_{r+s+t}^{T}
\end{array}\right]^{T} .
$$

(If $s$ or $t=1$, then there are no $W_{i}$ 's or $u_{i}$ 's in $A$, respectively.) Then

$$
B=A\left[\begin{array}{cc}
1 & 0 \\
0 & -1
\end{array}\right] A^{T}
$$

has rank 2. (By Lemma $6.1 B$ has partial inertia $(1,1)$. This is necessary since $B$ could not have zero entries on the diagonal as desired if it were positive semi-definite.) With the reflection matrix in the product, both $x$ and $y$ are "self orthogonal" but not 
orthogonal to each other, or any of the $u_{i}$ or $v_{i}$. Also, $u_{i}$ and $v_{i}$ are orthogonal with the reflection but not orthogonal to themselves, or any other $u_{j}$ or $v_{j}$, by our choices of the $\theta_{i}$ 's. Thus we see the $U_{i}$ 's correspond to the $K_{m_{i}, n_{i}}$ 's, $X$ corresponds to the $K_{2}$ with zeros on the diagonal, the $W_{i}$ 's correspond to the $K_{2}$ 's with nonzero diagonal entries, $y$ corresponds to the $K_{1}$ with zero diagonal, and the $u_{i}$ 's correspond to the $K_{1}$ 's with nonzero diagonal entries. So $B \in \mathcal{S}(G)$, and hence in $\mathscr{M} \mathscr{R}(G)$. We have shown that any diagonal entry corresponding to a $K_{1}$ or $K_{2}$ can be zero (though not all at once). We have similarly shown that if $s>1$ and $t>1$, any corresponding diagonal entry can be nonzero and, by replacing $X$ with $W_{r+1}$ and $y$ with $u_{r+s+1}$, all can be nonzero at once. This replacement argument shows that we have proven the case $s$ or $t=1$. Thus the vertices corresponding to the $K_{2}$ 's and $K_{1}$ 's are neutral.

In the case that $k \geq 3$ and $s, t \geq 1$, the vertices corresponding to $K_{k}$ are nil. Since only vectors of the form $x$ and $y$ will give zero diagonals in the product above, we can only create matrices with one $K_{2}$ or one $K_{1}$ with zero diagonals, not both. Let $Z$ be a $k \times 2$ matrix with $k$ rows of $x$, and construct $A$ as before, but insert $Z$ at the beginning and use either one of $X$ and $y$. The same argument shows that the vertices corresponding to the $K_{2}$ 's and $K_{1}$ 's are neutral.

If both $s, t=0$, we have nothing to show. If one of $s$ or $t=0$, leave $X$ or $y$, respectively, out of $A$. Note that if $s$ or $t=0$, the $W_{i}$ 's or $u_{i}$ 's, respectively, are automatically not included. Construct $A$ as such, with $Z$ included or excluded depending on whether $k \geq 3$ or $k=0$. The arguments for the cases above show that the vertices corresponding to whichever of the $K_{2}$ 's or $K_{1}$ 's that are present are neutral.

The preceding results can be summarized as follows:

TheOrem 5.7. Let $G$ be a connected graph with $\operatorname{mr}(G)=2$ and write $G=$ $\left(K_{k} \cup K_{\ell} \cup K_{m_{1}, n_{1}} \cup \cdots \cup K_{m_{r}, n_{r}} \cup s K_{2} \cup t K_{1}\right)^{c}$, where $k, \ell \in\{0,3,4,5, \ldots\}, s, t \geq 0$, and none of the $K_{m_{i}, n_{i}}$ are $K_{2}$ 's or $K_{1}$ 's. Then the vertices of $G$ corresponding to

- $K_{k}$ and $K_{\ell}$ are nil vertices.

- the $K_{m_{i}, n_{i}}$ 's are nonzero vertices.

- the $K_{2}$ 's and $K_{1}$ 's are nonzero vertices if $k, \ell \geq 3$ and neutral vertices otherwise.

6. A solution for the inverse eigenvalue problem for graphs whose minimum rank is two. In this section, we will use our results on the inertia sets of connected minimum rank 2 graphs and our results on the structure of these minimum rank matrices to investigate the Inverse Eigenvalue Problem for graphs whose minimum rank is 2 . We will solve this problem in the case of minimum rank matrices for these graphs. Our result resembles a result in [1] where the authors show that the 
multiplicity list alone is not sufficient to determine the solution to the inverse eigenvalue problem for a tree. Certain magnitudinal issues are critical. Similarly, we show that the inertia set alone of a minimum rank two graph does not give all restrictions on possible eigenvalues of the graph. In three cases there are additional conditions.

We include some standard results from matrix theory that we will use extensively.

Lemma 6.1. A symmetric $n \times n$ matrix $M$ has partial inertia $(r, s)$ with $r+s=k$ if and only if $M$ has a factorization

$$
M=A\left[\begin{array}{cccccc}
1 & & & & & \\
& \ddots & & & 0 & \\
& & 1 & & & \\
& & & -1 & & \\
& 0 & & & \ddots & \\
& & & & & -1
\end{array}\right] A^{T},
$$

where $A$ is an $n \times k$ matrix, and we have 1 occurring $r$ times on the diagonal, and -1 occurring s times.

If $M$ is positive semidefinite, then Lemma 6.1 simply states that $M$ is the Gram matrix of some set of vectors, $M=A A^{T}$.

Lemma 6.2. [9, Theorem 1.3.20] Suppose $A$ is $m \times n$ and $B n \times m$ with $m \leq n$. Then $B A$ has the same eigenvalues as $A B$, counting multiplicity, together with an additional $n-m$ eigenvalues equal to 0 .

Our strategy will be to look at several cases based on the characterization of minimum rank 2 graphs in Theorem 4.3 and each possible inertia, and figure out the general structure of $A$ in the factorization from Lemma 6.1 to guarantee the matrix corresponds to the graph. Then we will multiply in the reverse order, to attain a smaller matrix (in our case $2 \times 2$ ) whose eigenvalues we can compute easily.

THEOREM 6.3. Let $G$ be a connected graph whose minimum rank is 2. Then we have the following restrictions in the Inverse Eigenvalue Problem:

- If $G=\left(K_{1} \cup K_{m, n}\right)^{c}, m, n \geq 1$, (that is, $G$ is a vertex sum of two cliques), then a rank minimizing matrix for $G$ cannot have a nonzero eigenvalue of multiplicity two.

- If $G=\left(K_{k} \cup K_{\ell}\right)^{c}=K_{k, \ell}, k, \ell \geq 3$, then the two nonzero eigenvalues of a rank minimizing matrix for $G$ must sum to 0 .

- If $G=\left(K_{k} \cup K_{\ell} \cup K_{1}\right)^{c}=K_{k, \ell} \vee K_{1}, k, \ell \geq 3$, then the two nonzero eigenvalues of a rank minimizing matrix for $G$ cannot sum to 0 .

Any two nonzero eigenvalues not ruled out by the inertia set or the restrictions above 
can be attained by a rank minimizing matrix for a graph whose minimum rank is 2.

\section{Proof.}

I. First we will consider the positive semidefinite case (partial inertia $(2,0)$ ). By Theorem 3.3, any connected graph with minimum positive semidefinite rank 2 is the complement of the union of complete bipartite graphs. In our computations, we will consider $K_{1}$ separately from other complete bipartite graphs.

Case 1. First let $G=\left(p K_{1} \cup K_{m_{1}, n_{1}} \cup \cdots \cup K_{m_{r}, n_{r}}\right)^{c}, r \geq 1, p \geq 2, m_{i}, n_{i} \geq 1$ (if $r=0$ then $G$ is complete and does not have minimum rank 2). First consider the case $r \geq 2$. Let

$$
\begin{aligned}
z & =\left[\frac{1}{\sqrt{p}}, \frac{a}{\sqrt{p}}\right], & w & =\left[\frac{1}{\sqrt{p}},-\frac{(p-1) a}{\sqrt{p}}\right], \\
u_{1} & =\left[\frac{\sqrt{x}}{\sqrt{m_{1}}}, 0\right], & v_{1} & =\left[0, \frac{\sqrt{y}}{\sqrt{n_{1}}}\right], \\
u_{i} & =\left[\frac{\cos \theta_{i}}{\sqrt{(r-1) m_{i}}}, \frac{\sin \theta_{i}}{\sqrt{(r-1) m_{i}}}\right], & v_{i} & =\left[-\frac{\sin \theta_{i}}{\sqrt{(r-1) n_{i}}}, \frac{\cos \theta_{i}}{\sqrt{(r-1) n_{i}}}\right] \text { for } i=2, \ldots, r,
\end{aligned}
$$

where $x, y>0,0<\theta_{2}<\cdots<\theta_{r}<\frac{\pi}{4}$, and $a$ is chosen so that $z$ and $w$ are not orthogonal to any of the other vectors (any $a>1$ will guarantee $z$ and $w$ are not orthogonal, and that $z$ is not orthogonal to any of the $u_{i}$ or $v_{i}$; then choose $a$ so that $w$ is not orthogonal to any of the $u_{i}$ and $v_{i}$ ). Also note that $u_{i}$ and $v_{i}$ are orthogonal to each other, but to none of the other vectors. Let $Z$ be the $(p-1) \times 2$ matrix with the vector $z$ in each row, and $U_{i}$ the $\left(m_{i}+n_{i}\right) \times 2$ matrix with $u_{i}$ in the first $m_{i}$ rows and $v_{i}$ in the last $n_{i}$ rows.

Let

$$
A=\left[\begin{array}{c}
Z \\
w \\
U_{1} \\
\vdots \\
U_{r}
\end{array}\right] .
$$

Then $A A^{T} \in \mathcal{S}(G)$, has rank 2, and is positive semidefinite. Multiplying in the other order, we get

$$
A^{T} A=\left[\begin{array}{cc}
2+x & 0 \\
0 & 1+(p-1) a^{2}+y
\end{array}\right] .
$$

With the appropriate choice of $x$ and $y$, it is clear that, after scaling, we can attain any two positive eigenvalues we wish (including a positive eigenvalue of multiplicity 
2 ). Note also that if $r=1$, simply leave out $U_{2}, \ldots, U_{r}$ from $A$, then

$$
A^{T} A=\left[\begin{array}{cc}
1+x & 0 \\
0 & (p-1) a^{2}+y
\end{array}\right]
$$

and the same argument works.

Case 2. Now consider $p=0, G=\left(K_{m_{1}, n_{1}} \cup \cdots \cup K_{m_{r}, n_{r}}\right)^{c}, r \geq 2$ (if $r=1$ we get a disconnected graph which we will not consider). For this case, keep $u_{i}, v_{i}$ the same as they were before, and let

$$
A=\left[\begin{array}{c}
U_{1} \\
\vdots \\
U_{r}
\end{array}\right]
$$

Then $A A^{T} \in \mathcal{S}(G)$, and

$$
A^{T} A=\left[\begin{array}{cc}
1+x & 0 \\
0 & 1+y
\end{array}\right] .
$$

After scaling, we can clearly attain any two positive eigenvalues we wish with this.

Case 3. Now suppose $p=1$ and $r \geq 2$. Let

$$
\begin{aligned}
& z=\left[\frac{3}{2},-1\right] \text {, } \\
& u_{1}=\left[\frac{\sqrt{x}}{\sqrt{m_{1}}}, 0\right], \quad v_{1}=\left[0, \frac{\sqrt{y}}{\sqrt{n_{1}}}\right] \\
& u_{2}=\left[\frac{\sqrt{2}}{\sqrt{m_{2}}}, \frac{\sqrt{2}}{\sqrt{m_{2}}}\right], \quad v_{2}=\left[-\frac{\sqrt{2}}{2 \sqrt{n_{2}}}, \frac{\sqrt{2}}{2 \sqrt{n_{2}}}\right] \\
& u_{i}=\left[\frac{\cos \theta_{i}}{\sqrt{(r-2) m_{i}}}, \frac{\sin \theta_{i}}{\sqrt{(r-2) m_{i}}}\right], \quad v_{i}=\left[-\frac{\sin \theta_{i}}{\sqrt{(r-2) n_{i}}}, \frac{\cos \theta_{i}}{\sqrt{(r-2) n_{i}}}\right] \text { for } i=3, \ldots, r
\end{aligned}
$$

and let

$$
A=\left[\begin{array}{c}
z \\
U_{1} \\
\vdots \\
U_{r}
\end{array}\right]
$$

where $U_{1}, \ldots, U_{r}$ are defined in terms of these $u_{i}$ and $v_{i}$ as before.

Then $A A^{T} \in \mathcal{S}(G)$ and for $r \geq 3$,

$$
A^{T} A=\left[\begin{array}{cc}
\frac{23}{4}+x & 0 \\
0 & \frac{9}{2}+y
\end{array}\right] .
$$


It is clear that after scaling, the appropriate choice of $x$ and $y$ yields any two positive eigenvalues we wish (including a multiplicity 2 eigenvalue). Note that if $r=2$, simply leave out $U_{3}, \ldots, U_{r}$. Then

$$
A^{T} A=\left[\begin{array}{cc}
\frac{19}{4}+x & 0 \\
0 & \frac{7}{2}+y
\end{array}\right]
$$

and the same argument works.

Case 4. Now suppose $p=1$ and $r=1$. Any rank 2 positive semidefinite matrix in $\mathcal{S}(G)$ can be factored $A A^{T}$, where $A$ is $n \times 2$, and the $m_{1}$ rows corresponding to the first $m_{1}$ vertices in $K_{m_{1}, n_{1}}$ in the complement must be orthogonal to the $n_{1}$ rows corresponding to the other $n_{1}$ vertices in the $K_{m_{1}, n_{1}}$. For any such matrix, multiplication by a $2 \times 2$ rotation matrix on the right puts those rows in "standard position," that is, where rows have the form $(a, 0)$ and $(0, b)$. So without loss of generality, we may assume that

$$
A=\left[\begin{array}{cc}
x & y \\
a_{1} & 0 \\
\vdots & \\
a_{m_{1}} & 0 \\
0 & b_{1} \\
\vdots & \\
0 & b_{n_{1}}
\end{array}\right]
$$

and thus

$$
A^{T} A=\left[\begin{array}{cc}
x^{2}+\sum a_{i}^{2} & x y \\
x y & y^{2}+\sum b_{i}^{2}
\end{array}\right] .
$$

If this has an eigenvalue of multiplicity 2 , then it is similar to a multiple of the identity, and thus is a multiple of the identity. Therefore, $x y=0$, which implies that either $x=0$ or $y=0$, but that would not correspond to the graph that we are considering. Thus, a positive eigenvalue of multiplicity 2 is impossible in this case.

To get any other possibility, just take

$$
z=[\sqrt{x}, \sqrt{x}], u_{1}=\left[\frac{1}{\sqrt{m_{1}}}, 0\right], v_{1}=\left[0, \frac{1}{\sqrt{n_{1}}}\right],
$$

and set $A=\left[\begin{array}{c}z \\ U_{1}\end{array}\right]$. Then $A A^{T} \in \mathcal{S}(G)$ and

$$
A^{T} A=\left[\begin{array}{cc}
x+1 & x \\
x & x+1
\end{array}\right]=\left[\begin{array}{ll}
x & x \\
x & x
\end{array}\right]+I .
$$


The matrix $\left[\begin{array}{ll}x & x \\ x & x\end{array}\right]$ has rank 1 and trace $2 x$, so its eigenvalues are 0 and $2 x$. Thus the eigenvalues of $A^{T} A$ are 1 and $1+2 x$. It is clear that, after scaling, we can attain any two distinct, positive eigenvalues that we wish.

This case gives the first restriction in the statement of the theorem.

II. Now we will consider the inertia $(1,1)$ case. With Lemma 6.1 in mind, we define an indefinite inner product $\langle\cdot, \cdot\rangle$ on $\mathbb{R}^{2}$ by

$$
\langle a, b\rangle=a\left[\begin{array}{cc}
1 & 0 \\
0 & -1
\end{array}\right] b^{T} .
$$

Again, $K_{1}$ will be considered separately from other complete bipartite graphs.

First let $G=\left(K_{k} \cup K_{\ell} \cup K_{m_{1}, n_{1}} \cup \cdots \cup K_{m_{r}, n_{r}}\right)^{c}$, where $k, \ell \geq 3, r \geq 2$, and $n_{i}, m_{i} \geq 1$ for all $i$ (so no $K_{1}$ 's are in the complement, but we do allow $K_{2}$ 's).

Let $s>0, t \geq 0$, and let

$$
\begin{array}{ll}
x=\left[\frac{\sqrt{s}}{\sqrt{k}}, \frac{\sqrt{s}}{\sqrt{k}}\right], & y=\left[\frac{\sqrt{s}}{\sqrt{\ell}},-\frac{\sqrt{s}}{\sqrt{\ell}}\right], \\
u_{1}=\left[\frac{\sqrt{t+s}}{\sqrt{m_{1}}}, 0\right], & v_{1}=\left[0, \frac{\sqrt{s}}{\sqrt{n_{1}}}\right], \\
u_{i}=\left[\frac{\sqrt{s} \cos \theta_{i}}{\sqrt{(r-1) m_{i}}}, \frac{\sqrt{s} \sin \theta_{i}}{\sqrt{(r-1) m_{i}}}\right], & v_{i}=\left[\frac{\sqrt{s} \sin \theta_{i}}{\sqrt{(r-1) n_{i}}}, \frac{\sqrt{s} \cos \theta_{i}}{\sqrt{(r-1) n_{i}}}\right] \text { for } i=2, \ldots, r,
\end{array}
$$

where $0<\theta_{2}<\theta_{3}<\cdots<\theta_{r}<\frac{\pi}{4}$. Define $X$ to be the $k \times 2$ matrix with the vector $x$ in each row, $Y$ to be the $\ell \times 2$ matrix with $y$ in each row, and $U_{i}$ to be the $\left(m_{i}+n_{i}\right) \times 2$ matrix with the vector $u_{i}$ in the first $m_{i}$ rows, and $v_{i}$ in the last $n_{i}$ rows. Notice that both $x$ and $y$ are "self orthogonal" under our new inner product, but are not orthogonal to each other, or any of the $u_{i}$ or $v_{i}$. Also, $u_{i}$ and $v_{i}$ are orthogonal under this inner product, but are not orthogonal to themselves, or any other $u_{j}$ or $v_{j}$, by our choices of $\theta_{i}$.

Let

$$
A=\left[\begin{array}{lllll}
X^{T} & Y^{T} & U_{1}^{T} & \cdots & U_{r}^{T}
\end{array}\right]^{T}
$$

Then the matrix

$$
A\left[\begin{array}{cc}
1 & 0 \\
0 & -1
\end{array}\right] A^{T} \in \mathcal{S}(G)
$$

and has rank 2 and partial inertia $(1,1)$. Multiplying in the other order, we get

$$
\left[\begin{array}{cc}
1 & 0 \\
0 & -1
\end{array}\right] A^{T} A=\left[\begin{array}{cc}
2 s+(t+s)+s & 2 s \sum_{i=2}^{r} \frac{\cos \theta_{i} \sin \theta_{i}}{r-1} \\
-2 s \sum_{i=2}^{r} \frac{\cos \theta_{i} \sin \theta_{i}}{r-1} & -(2 s+s+s)
\end{array}\right]=\left[\begin{array}{cc}
t+4 s & 2 s \alpha \\
-2 s \alpha & -4 s
\end{array}\right],
$$


where $\alpha=\sum_{i=2}^{r} \frac{\cos \theta_{i} \sin \theta_{i}}{r-1}$. Note in particular that $\alpha<1$. This matrix has the same nonzero eigenvalues. The trace is $t$, and the determinant is $(t+4 s)(-4 s)+4 s^{2} \alpha^{2}=$ $-4 s t-16 s^{2}+4 s^{2} \alpha^{2}=-4 s t-s^{2}\left(16-4 \alpha^{2}\right)$. Thus the characteristic polynomial is

$$
\lambda^{2}-t \lambda-4\left(s t+s^{2}\left(4-\alpha^{2}\right)\right)
$$

and the eigenvalues are

$$
\frac{1}{2}\left(t \pm \sqrt{t^{2}+16\left(s t+s^{2}\left(4-\alpha^{2}\right)\right)}\right) .
$$

Then given $\lambda_{1}>0$ and $\lambda_{2}<0$ where, without loss of generality, $\lambda_{1} \geq\left|\lambda_{2}\right|$, let $t=\lambda_{1}+\lambda_{2}$. Then since $\lambda_{2}<0, \lambda_{1}-\lambda_{2}>t$. Notice that for $s \in(0, \infty)$, the range of $\sqrt{t^{2}+4\left(4 s t+s^{2}\left(16-4 \alpha^{2}\right)\right)}$ is $(t, \infty)$ (since $t \geq 0$ and $\alpha<1$ ), so choose $s$ so that $\lambda_{1}-\lambda_{2}=\sqrt{t^{2}+4\left(4 s t+s^{2}\left(16-4 \alpha^{2}\right)\right)}$. This choice of $t$ and $s$ give $\lambda_{1}$ and $\lambda_{2}$ as eigenvalues.

In the following table, we will look at several different cases using the above idea. We will still take $G=\left(K_{k} \cup K_{\ell} \cup p K_{1} \cup K_{m_{1}, n_{1}} \cup \cdots \cup K_{m_{r}, n_{r}}\right)^{c}$ but we will look at cases where some of the graphs are possibly missing. We will still assume that $n_{i}, m_{i} \geq 1$, so that none of those complete bipartite graphs are $K_{1}$, and $K_{1}$ will be considered separately. As a convention for convenience, if either of $k$ or $\ell$ is 0 , that simply means that that complete graph does not occur in the complement. In all the cases we look at we will take $k$ and $\ell$ either to be 0 or at least 3 . In the table, the first column describes the graph $G$ by stating which graphs are in the complement, the second column gives the matrix $A$ (with $X, Y$, and $U_{i}$ as above) that will guarantee $A\left[\begin{array}{cc}1 & 0 \\ 0 & -1\end{array}\right] A^{T} \in \mathcal{S}(G)$, the third column shows the matrix we get when we multiply in the other order, and the last column the eigenvalues of this matrix. We repeat the case above to give a complete reference.

For $u>0$ (and we still have $t \geq 0$ and $s>0$ ), define the vectors

$$
\begin{array}{ll}
z=\left[\frac{\sqrt{2 s}}{\sqrt{p}}, \frac{\sqrt{s}}{\sqrt{2 p}}\right], \quad z^{\prime}=\left[\frac{1}{\sqrt{p-1}},-\frac{\sqrt{u}}{\sqrt{p-1}}\right], \\
w=\left[\frac{\sqrt{u}}{\sqrt{p}}, 0\right], \quad w^{\prime}=[\sqrt{u}, 1], \\
v_{1}^{\prime}=\left[0, \frac{\sqrt{5 s}}{\sqrt{2 n_{1}}}\right],
\end{array}
$$

Define the matrix $Z$ to be the $p \times 2$ matrix with $z$ in all its rows, $Z^{\prime}$ the $(p-1) \times 2$ matrix with $z^{\prime}$ in all its rows, $W$ the $p \times 2$ matrix with $w$ in all its rows, and $U_{1}^{\prime}$ the matrix with $u_{1}$ in the first $m_{1}$ rows and $v_{1}^{\prime}$ in the last $n_{1}$ rows. We need $z$ not orthogonal (with respect to the inner product defined at the beginning of II) to any of the $u_{i}$ or $v_{i}$. To do so we only need $\theta_{i} \neq \arctan \frac{1}{2}$ for all $i$. 


\begin{tabular}{|c|c|c|c|c|c|}
\hline & $G$ & $A$ & {$\left[\begin{array}{cc}1 & 0 \\
0 & -1\end{array}\right]$} & $A^{T} A$ & Eigenvalues \\
\hline 1 & $\begin{array}{l}p=0 \\
k, \ell \geq 3 \\
r \geq 2\end{array}$ & {$\left[\begin{array}{c}X \\
Y \\
U_{1} \\
\vdots \\
U_{r}\end{array}\right]$} & {$\left[\begin{array}{l}t+4 s \\
-2 s \alpha\end{array}\right.$} & $\left.\begin{array}{l}2 s \alpha \\
-4 s\end{array}\right]$ & $\frac{1}{2}\left(t \pm \sqrt{t^{2}+16\left(s t+s^{2}\left(4-\alpha^{2}\right)\right)}\right)$ \\
\hline 2 & $\begin{array}{l}p=0 \\
k \geq 3 \\
\ell=0 \\
r \geq 2\end{array}$ & {$\left[\begin{array}{c}X \\
U_{1} \\
\vdots \\
U_{r}\end{array}\right]$} & {$\left[\begin{array}{c}t+3 s \\
-s-2 s \alpha\end{array}\right.$} & $\left.\begin{array}{cc} & s+2 s \alpha \\
\alpha & -3 s\end{array}\right]$ & $\frac{1}{2}\left(t \pm \sqrt{t^{2}+4\left(3 s t+4 s^{2}\left(2-\alpha-\alpha^{2}\right)\right)}\right)$ \\
\hline 3 & $\begin{array}{l}p=0 \\
k \geq 3 \\
\ell=0 \\
r=1\end{array}$ & {$\left[\begin{array}{c}X \\
U_{1}\end{array}\right]$} & {$\left[\begin{array}{c}t+2 s \\
-s\end{array}\right.$} & $\left.\begin{array}{c}s \\
-2 s\end{array}\right]$ & $\frac{1}{2}\left(t \pm \sqrt{t^{2}+8 s t+12 s^{2}}\right)$ \\
\hline 4 & $\begin{array}{l}p=0 \\
k, \ell=0 \\
r \geq 2\end{array}$ & {$\left[\begin{array}{c}U_{1} \\
\vdots \\
U_{r}\end{array}\right]$} & {$\left[\begin{array}{c}t+2 s \\
-2 s \alpha\end{array}\right.$} & $\left.\begin{array}{l}2 s \alpha \\
-2 s\end{array}\right]$ & $\frac{1}{2}\left(t \pm \sqrt{t^{2}+8\left(t s+2 s^{2}\left(1-\alpha^{2}\right)\right)}\right)$ \\
\hline 5 & $\begin{array}{l}p=0 \\
k, \ell \geq 3 \\
r=1\end{array}$ & {$\left[\begin{array}{c}X \\
Y \\
U_{1}\end{array}\right]$} & {$\left[\begin{array}{c}t+3 s \\
0\end{array}\right.$} & $\left.\begin{array}{c}0 \\
-3 s\end{array}\right]$ & $t+3 s,-3 s$ \\
\hline 6 & $\begin{array}{l}p=0 \\
k, \ell \geq 3 \\
r=0\end{array}$ & {$\left[\begin{array}{l}X \\
Y\end{array}\right]$} & {$\left[\begin{array}{cc}2 s & 0 \\
0 & -2 s\end{array}\right.$} & & $2 s,-2 s$ \\
\hline 7 & $\begin{array}{l}p \geq 1 \\
k, \ell=0 \\
r \geq 2\end{array}$ & {$\left[\begin{array}{c}Z \\
U_{1}^{\prime} \\
U_{2} \\
\vdots \\
U_{r}\end{array}\right]$} & {$\left[\begin{array}{c}t+4 s \\
-s-2 s c\end{array}\right.$} & $\left.\begin{array}{cc} & s+2 s \alpha \\
\alpha & -4 s\end{array}\right]$ & $\frac{1}{2}\left(t \pm \sqrt{t^{2}+4\left(4 s t+s^{2}\left(15-4 \alpha-4 \alpha^{2}\right)\right)}\right)$ \\
\hline 8 & $\begin{array}{l}p \geq 1 \\
k, \ell=0 \\
r=1\end{array}$ & {$\left[\begin{array}{c}Z \\
U_{1}^{\prime}\end{array}\right]$} & {$\left[\begin{array}{c}t+3 s \\
-s\end{array}\right.$} & $\left.\begin{array}{c}s \\
-3 s\end{array}\right]$ & $\frac{1}{2}\left(t \pm \sqrt{t^{2}+12 t s+32 s^{2}}\right)$ \\
\hline
\end{tabular}




\begin{tabular}{|c|c|c|c|c|c|}
\hline & $G$ & $A$ & {$\left[\begin{array}{cc}1 & 0 \\
0 & -1\end{array}\right]$} & $A^{T} A$ & Eigenvalues \\
\hline 9 & $\begin{array}{l}p \geq 1 \\
k, \ell \geq 3 \\
r \geq 2\end{array}$ & {$\left[\begin{array}{c}X \\
Y \\
Z \\
U_{1}^{\prime} \\
U_{2} \\
\vdots \\
U_{r}\end{array}\right]$} & {$\left[\begin{array}{r}t+6 s \\
-s-2 s\end{array}\right.$} & $\left.\begin{array}{cc}s+2 s \alpha \\
s \alpha & -6 s\end{array}\right]$ & $\frac{1}{2}\left(t \pm \sqrt{t^{2}+4\left(6 t s+s^{2}\left(35-4 \alpha-4 \alpha^{2}\right)\right)}\right)$ \\
\hline 10 & $\begin{array}{l}p \geq 1 \\
k, \ell \geq 3 \\
r=1\end{array}$ & {$\left[\begin{array}{c}X \\
Y \\
Z \\
U_{1}^{\prime}\end{array}\right]$} & {$\left[\begin{array}{c}t+5 s \\
-s\end{array}\right.$} & $\left.\begin{array}{c}s \\
-5 s\end{array}\right]$ & $\frac{1}{2}\left(t \pm \sqrt{t^{2}+4\left(5 t s+24 s^{2}\right)}\right)$ \\
\hline 11 & $\begin{array}{l}p \geq 1 \\
k \geq 3 \\
\ell=0 \\
r \geq 2\end{array}$ & {$\left[\begin{array}{c}X \\
Z \\
U_{1}^{\prime} \\
U_{2} \\
\vdots \\
U_{r}\end{array}\right]$} & {$\left[\begin{array}{r}t+5 s \\
-2 s-2\end{array}\right.$} & $\left.\begin{array}{lc}s & 2 s+2 s \alpha \\
2 s \alpha & -5 s\end{array}\right]$ & $\frac{1}{2}\left(t \pm \sqrt{t^{2}+4\left(5 s t+s^{2}\left(21-8 \alpha-4 \alpha^{2}\right)\right)}\right)$ \\
\hline 12 & $\begin{array}{l}p \geq 1 \\
k \geq 3 \\
\ell=0 \\
r=1\end{array}$ & {$\left[\begin{array}{c}X \\
Z \\
U_{1}^{\prime}\end{array}\right]$} & {$\left[\begin{array}{c}t+4 s \\
-2 s\end{array}\right.$} & $\left.\begin{array}{c}2 s \\
-4 s\end{array}\right]$ & $\frac{1}{2}\left(t \pm \sqrt{t^{2}+4\left(4 s t+12 s^{2}\right)}\right)$ \\
\hline 13 & $\begin{array}{l}p \geq 2 \\
k, \ell \geq 3 \\
r=0\end{array}$ & {$\left[\begin{array}{c}X \\
Y \\
W\end{array}\right]$} & {$\left[\begin{array}{c}2 s+u \\
0\end{array}\right.$} & $\left.\begin{array}{c}0 \\
-2 s\end{array}\right]$ & $2 s+u,-2 s$ \\
\hline 14 & $\begin{array}{l}p=1 \\
k, \ell \geq 3 \\
r=0\end{array}$ & {$\left[\begin{array}{l}X \\
Y \\
w\end{array}\right]$} & {$\left[\begin{array}{c}2 s+u \\
0\end{array}\right.$} & $\left.\begin{array}{c}0 \\
-2 s\end{array}\right]$ & $2 s+u,-2 s$ \\
\hline 15 & $\begin{array}{l}p \geq 1 \\
k \geq 3 \\
\ell=0 \\
r=0\end{array}$ & {$\left[\begin{array}{l}X \\
W\end{array}\right]$} & {$\left[\begin{array}{c}s+u \\
-s\end{array}\right.$} & $\left.\begin{array}{c}s \\
-s\end{array}\right]$ & $\frac{1}{2}\left(u \pm \sqrt{u^{2}+4 s u}\right)$ \\
\hline
\end{tabular}

In Cases 2-4 and 7-12, it is straightforward to check that the same argument that 
we used in Case 1 works to show that we can achieve any positive and any negative eigenvalue that we wish (recall that $\alpha<1$ ). In Case 5 , it is clear that the appropriate choice of $s>0$ and $t \geq 0$ gives any eigenvalues we want.

In Case 6, the matrix constructed has trace zero, but by Theorem 5.1, all of the diagonal entries must be zero, so this gives all possibilities for this case. This gives the second restriction in the statement of the theorem.

In Case 13, since $u>0$, this does not allow for eigenvalues that add to zero, but after scaling, gives any other eigenvalues we wish. To attain equal and opposite eigenvalues, take $A=\left[\begin{array}{c}X \\ Y \\ Z^{\prime} \\ w^{\prime}\end{array}\right]$. For this case take $u>1$ so that $z^{\prime}$ and $w^{\prime}$ are not orthogonal to $x$ or $y$. Then $A\left[\begin{array}{cc}1 & 0 \\ 0 & -1\end{array}\right] A^{T} \in \mathcal{S}(G)$ and multiplying in the other order gives

$$
\left[\begin{array}{cc}
2 s+1+u & 0 \\
0 & -2 s-1-u
\end{array}\right]
$$

which has trace 0 , so after scaling, we can achieve any equal and opposite eigenvalues.

In Case 14, since $u>0$, this does not allow for eigenvalues that add to zero, but after scaling, gives any other eigenvalues we wish. Note that by Theorem 5.7, each diagonal entry corresponding to a vertex in one of the complete graphs in the complement must be 0 , and the diagonal entry corresponding to $K_{1}$ in the complement cannot be 0 . Thus we have exactly one nonzero entry on the diagonal, so the trace cannot be 0 . Thus our construction gives all possibilities for this case. This is the final restriction in the statement of the theorem.

In Case 15, using an argument similar to the first case, we can attain any two eigenvalues of opposite sign we wish, as long as they do not add up to 0 (recall $u>0$ is the trace). However, for $p>1$, consider the matrix

$$
M=\left[\begin{array}{ccccccc}
p-1 & 1 & \ldots & 1 & \sqrt{p}-1 & \cdots & \sqrt{p}-1 \\
1 & -1 & \cdots & -1 & 1 & & 1 \\
\vdots & \vdots & \ddots & \vdots & \vdots & \cdots & \vdots \\
1 & -1 & \cdots & -1 & 1 & & 1 \\
\sqrt{p}-1 & 1 & \cdots & 1 & 0 & \cdots & 0 \\
\vdots & & \vdots & & & \ddots & \\
\sqrt{p}-1 & 1 & \cdots & 1 & 0 & \cdots & 0
\end{array}\right] \in \mathcal{S}(G) .
$$

This matrix has only 3 distinct rows, and multiplying the second row by $-(\sqrt{p}-1)$ 
and adding it to the first row gives $\sqrt{p}$ times the last row. Thus, $M$ has rank 2 , and has trace 0 by construction. Thus, it has one positive and one negative eigenvalue which are opposite. So by multiplying $M$ by the appropriate scalar, we can attain any two opposite eigenvalues we wish.

For $p=1$, just take the appropriate multiple of

$$
\left[\begin{array}{cccc}
0 & 1 & \cdots & 1 \\
1 & 0 & \cdots & 0 \\
\vdots & & \ddots & \\
1 & 0 & \cdots & 0
\end{array}\right]
$$

to attain equal and opposite eigenvalues.

By Theorem 4.3, we have covered every connected graph whose minimum rank is 2. $\square$

7. Conclusion. In summary, we have characterized all graphs $G$ with $\operatorname{mr}(G)=2$ and $\mathrm{mr}_{+}(G)=k$ for any $k$ and showed that $\mathrm{mr}_{+}(G)=\alpha(G)$ for such graphs. This characterization solves the inverse inertia problem for graphs whose minimum rank is two. We next considered the structure of rank minimizing matrices for these graphs and classified all the nil, nonzero, and neutral vertices. Lastly, we solved the inverse eigenvalue problem for rank minimizing matrices for graphs whose minimum rank is two.

A few related open questions are listed below.

Question 7.1. For a graph $G$ with $\operatorname{mr}(G) \neq 2$, how are $\operatorname{mr}_{+}(G)$ and $\alpha(G)$ related?

Question 7.2. For a graph $G$ when is a vertex $v$ a nil vertex? When is $v$ a nonzero vertex?

For more results about the above question, see [2].

Question 7.3. Given a graph $\mathrm{G}$ with $\operatorname{mr}(G)=2$ and $\operatorname{mr}_{+}(G)=k \neq 2$, what is the solution to the inverse eigenvalue problem for matrices in $\mathcal{S}(G)$ with inertia $(k, 0)$ ?

Question 7.4. Can the techniques used for the inverse eigenvalue problem in this paper be extended to graphs whose minimum rank is not two?

Acknowledgement We thank H. Tracy Hall for the idea of putting the rows in standard position in Case 4 of I in Section 6. 


\section{REFERENCES}

[1] F. Barioli and S. Fallat. On two conjectures regarding an inverse eigenvalue problem for acyclic symmetric matrices. Electronic Journal of Linear Algebra, 11:41-50, 2004.

[2] W. Barrett, S. Gibelyou, M. Kempton, N. Malloy, C. Nelson, W. Sexton, and J. Sinkovic. Minimum rank matrices and nil vertices. In preparation.

[3] W. Barrett, H. Tracy Hall, and R. Loewy. The inverse inertia problem for graphs: Cut vertices, trees, and a counterexample. Linear Algebra and its Applications, 431:1147-1191, 2009.

[4] W. Barrett, H. van der Holst, and R. Loewy. Graphs whose minimal rank is two. Electronic Journal of Linear Algebra, 11:258-280, 2004.

[5] W. Barrett, C. Jepsen, R. Lang, E. McHenry, C. Nelson, and K. Owens. Inertia sets for graphs on six or fewer vertices. Electronic Journal of Linear Algebra, 20:53-78, 2010.

[6] M. Booth, P. Hackney, B. Harris, C.R. Johnson, M. Lay, L.H. Mitchell, S.K. Narayan, A. Pascoe, K. Steinmetz, B.D. Sutton, and W. Wang. On the minimum rank among positive semidefinite matrices with a given graph. SIAM Journal on Matrix Analysis and Applications, $30: 2: 731-740,2008$.

[7] D.G. Corneil, H. Lerchs, and L. Stewart Burlingham. Complement reducible graphs. Discrete Applied Mathematics, 3:163-174, 1981.

[8] P. Hackney, B. Harris, M. Lay, L.H. Mitchell, S.K. Narayan, and A. Pascoe. Linearly independent vertices and minimum semidefinite rank. Linear Algebra and its Applications, 431:1105$1115,2009$.

[9] R.A. Horn and C.R. Johnson. Matrix Analysis. Cambridge University Press, New York, 1985.

[10] M. Kempton and J. Sinkovic. Minimum rank of outerplanar graphs. Linear Algebra and its Applications, to appear. 